\title{
A Study on Electric Power Smoothing System for Lead-Acid Battery of Stand-Alone Natural Energy Power System Using EDLC
}

\author{
Yan Jia Student Member (jiayan@cs.elec.mie-u.ac.jp) \\ Ryosuke Shibata Student Member (ryosuke111@hotmail.com) \\ Naoki Yamamura Member (yamamura@elec.mie-u.ac.jp) \\ Muneaki Ishida Member (ishida@elec.mie-u.ac.jp)
}

Keywords: lead-acid battery, Stand-alone Natural Energy Power System (SNEPS), prolonging of service life, Electric Double Layer Capacitor (EDLC), bi-directional compensation system, electric power smoothing

In Stand-alone Natural Energy Power System (SNEPS) with power energy-storage device, power fluctuation in short period is one of the main reasons that recharge cycle times increases and lead-acid battery early failure. Hence, this paper proposed a electric power smoothing method for lead-acid battery of SNEPS using bidirectional Buck/Boost converter and Electric Double Layer Capacitor (EDLC), in order to prolong the service life of lead-acid battery through suppressing the power fluctuation. The validity of proposed system is confirmed by frequency characteristics analysis result and experimental results of actual WTG power system. In current study, as a example, The frequency region of $0.5[\mathrm{~Hz}] \leq f_{h} \leq 3[\mathrm{~Hz}]$ is determined as the target frequency region of power fluctuation suppressing.

A smoothing precision is defined to evaluate the smoothing level of power fluctuation suppressing. Here, the output voltage of battery is approximated constant, the electric power smoothing is considered by considering the output current smoothing. As a result, the smoothing precision is given in (1). We purpose to gain the smoothing precision of smaller than $10 \%$.

$$
\begin{aligned}
& \text { Smoothing Precision } \\
& =\frac{\text { Current Fluctuation of Lead }- \text { acid Battery }}{\text { Current Fluctuation of Natural Energy Power Supply }}
\end{aligned}
$$

Fig. 1 shows the configuration of the proposed system. Where an EDLC is connected with lead-acid battery in parallel as the hybrid energy storage device, based on charge/discharge of EDLC, EDLC compensates the harmonic component of power fluctuation of short period by controlling the bi-directional converter, in order to supply smooth component to battery. A proportional \& integral (PI) controller is adopted to control and adjust the steady state error $\Delta i b\left(\Delta i b=i b_{\text {ref }}-i b\right)$ of reference and battery current tend to zero. The simulation result of stable operation of the proposed system is shown in Fig. 2. Fig. 2 shows the current fluctuation, is is suppressed and smoothed current, $i b$ is supplied to battery. However, the nonlinear phenomenon results in the characteristics analysis of system that using conventional perturbation analysis theory becomes complex and difficult. Hence, parameters that satisfy electric power smoothing output are selected in the stable region during system design using PSIM software and the effect trend-line.

Fig. 3 shows the calculation results and experimental results of frequency characteristic of the proposed system. The results satisfy the requirement of smoothing precision in the defined frequency target region. In addition, the validity of the proposed system is confirmed in actual stand-alone WTG power system too, the experimental results are shown in Fig. 4.

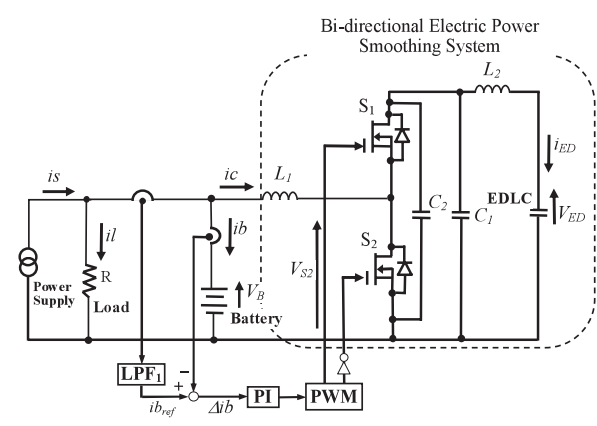

Fig. 1. Configuration of proposed electric power smoothing system $i s=10 \sin (6 \pi t)+10[\mathrm{~A}], S=2.2 \%$

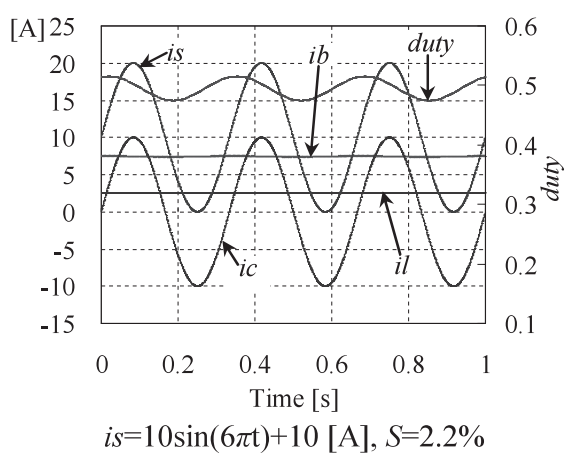

Fig. 2. Stable operation of proposed electric power smoothing system

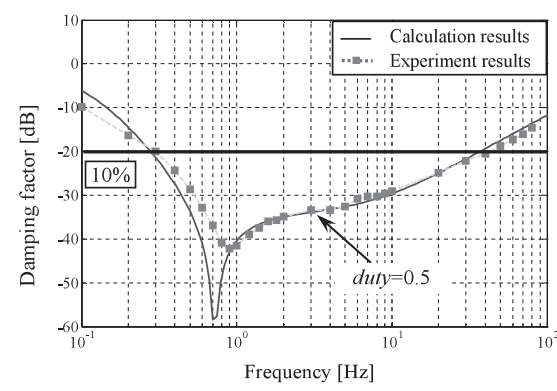

Fig. 3. Frequency characteristics

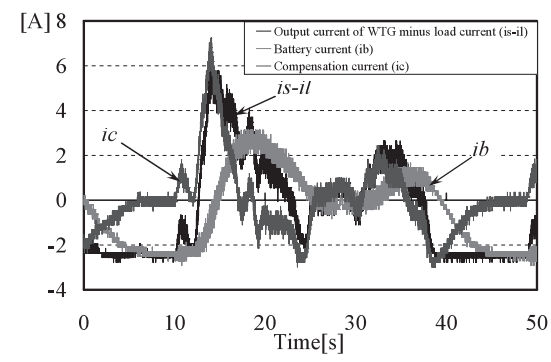

Fig. 4. An example of experimental results in actual stand-alone WTG power system with proposed system 


\title{
A Study on Electric Power Smoothing System for Lead-Acid Battery of Stand-Alone Natural Energy Power System Using EDLC
}

\author{
Yan Jia* Student Member \\ Ryosuke Shibata* Student Member \\ Naoki Yamamura* Member \\ Muneaki Ishida* Member
}

\begin{abstract}
To resolve energy shortage and global warming problem, renewable natural resource and its power system has been gradually generalizing. However, the power fluctuation suppressing in short period and the balance control of consumption and supply in long period are two of main problems that need to be resolved urgently in natural energy power system. In Stand-alone Natural Energy Power System (SNEPS) with power energy storage devices, power fluctuation in short period is one of the main reasons that recharge cycle times increase and lead-acid battery early failure. Hence, to prolong the service life of lead-acid battery and improve power quality through suppressing the power fluctuation, we proposed a method of electric power smoothing for lead-acid battery of SNEPS using bi-directional Buck/Boost converter and Electric Double Layer Capacitor (EDLC) in this paper. According to the test data of existing SNEPS, a power fluctuation condition is selected and as an example to analyze the validity of the proposed method. The analysis of frequency characteristics indicates the power fluctuation is suppressed a desired range in the target frequency region. The experimental results of confirmed the feasibility of the proposed system and the results well satisfy the requirement of system design.
\end{abstract}

Keywords: lead-acid battery, Stand-alone Natural Energy Power System (SNEPS), prolonging of service life, Electric Double Layer Capacitor (EDLC), bi-directional compensation system, electric power smoothing

\section{Introduction}

Recently, Stand-alone Natural Energy Power System (SNEPS) using wind, solar, etc. has become the main power supply method for the rural areas without grid access. The power fluctuation suppressing of short period and the demand and supply balance control of long period are two of main problems needed to be resolved urgently in natural energy power system ${ }^{(1)}$. Especially in the conventional standalone power system, the power fluctuation caused by erratic natural condition impacts the service life of energy storage device badly such as lead-acid battery. Hence, the target of our study is to prolong the service life of lead-acid battery through smoothing power fluctuation, focusing on the power fluctuation component of short period.

Energy storage device is the necessary part in SNEPS to ensure the stable power supply and improve power quality. Now, lead-acid battery is the most common energy storage device in SNEPS because of its higher capacity, the least initial cost and being readily available nearly everywhere in the world. However, lead-acid battery is an electro-chemical battery, it usually early failure due to some internal or exterior reason, e.g., the overheating caused by loss of inherent resistance during charging, the deterioration of electrodes and electrolyte due to repetition of charge/discharge, improper charging and saving method, and so on. Not only the internal

\footnotetext{
* Dept. of E. E. Eng., Faculty of Eng., Mie University

1577, Kurimamachiya-cho, Tsu 514-8507
}

reason but also the exterior charger, in the final analysis, the main reason of early deterioration is the recharge cycle times increasing. When the fluctuant power is supplied to lead-acid battery directly, recharge cycle times of short period increases greatly which results in earlier failure of leadacid battery. In addition, renewable natural generator has the weak point of impossibility to control their output themselves which will cause some problem with respect to power quality such as voltage and frequency fluctuation ${ }^{(2)}$.

Hence, an electric power smoothing system is proposed in this paper. Where an EDLC ${ }^{(3)(4)}$ connects with lead-acid battery in parallel and as the hybrid energy-storage device of SNEPS, and based on charge/discharge of EDLC, EDLC compensates the harmonic component of power fluctuation of short period by controlling a bi-directional Buck/Boost converter $^{(5)}$, in order to supply smoothed component of power supply to lead-acid battery. The validity of the proposed electric power smoothing system is verified by simulation in PSIM and actual experimental test.

\section{Power Fluctuation Suppressing}

2.1 Output Characteristics of Natural Power System

The conventional SNEPS is shown as Fig. 1. Usually, the lead-acid battery connects with power source consisting of PV (Photovoltaic) panel and WTG (Wind Turbine Generator) through a charge/discharge controller. Fig. 2(a) is an example of WTG output. Fig. 2(a) shows the output of natural energy power system changing frequently not only with the seasons in long period but also with seconds in short period. When 


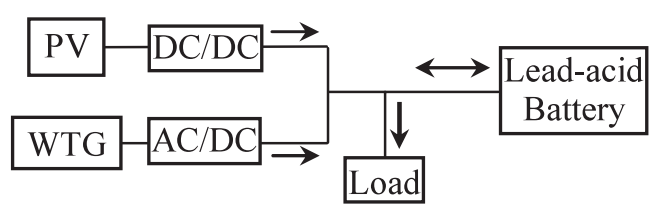

Fig. 1. Conventional stand-alone natural power system

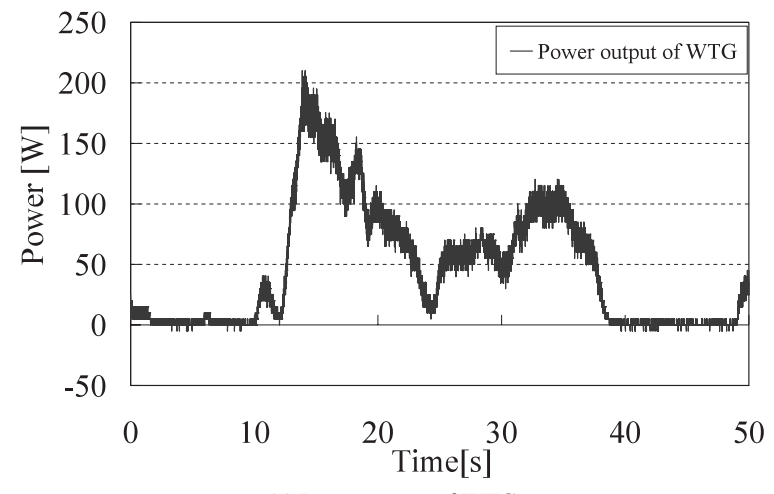

(a) Power output of WTG

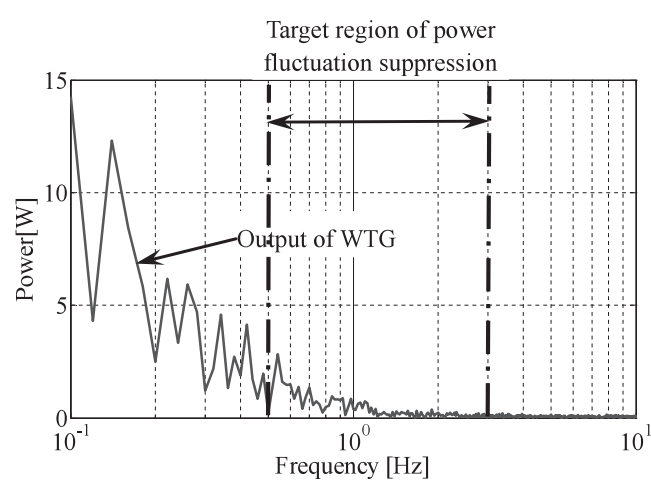

(b) Frequency spectrum of output of WTG

Fig. 2. An example of output of stand-alone WTG

the balance point of consumption and supply is in the short period of power fluctuation, the charge/discharge controller can not prevent the impact of power fluctuation on recycle times of lead-acid battery, which results in the lead-acid battery prone early failure. Hence, the power fluctuation suppressing of short period is necessary to prolong the service of lead-acid battery in SNEPS.

2.2 Power Fluctuation Suppressing In lower frequency region, the power fluctuation suppressing requires the bigger capacity of EDLC, hence, we select $0.5[\mathrm{~Hz}]$ as the lower limit frequency of the power fluctuation suppressing. In addition, because the lead-acid battery is a chemical storage device, when the frequency of input power so high compared with chemical reaction speed, we can consider that the high frequency component of the power fluctuation hardly affects charge/discharge of lead-acid battery. In the case, we focus the power fluctuation suppressing that the fluctuation frequency is smaller than $100[\mathrm{~Hz}]$. That is, generally, we study the power fluctuation suppression that the fluctuation frequency region is $0.5[\mathrm{~Hz}] \leq f_{h} \leq 100[\mathrm{~Hz}]$. To verify the validity of the proposed electric power smoothing compensation system, the target region of power fluctuation suppression is determined by analyzing the frequency spectrum of the WTG output power ${ }^{(6)}$. Fig. 2(b) shows the frequency spectrum of the WTG output power which shows in Fig. 2(a). Based on the existing data, we consider the frequency region of $0.5[\mathrm{~Hz}] \leq f_{h} \leq 3[\mathrm{~Hz}]$ as an example of the target region of the power fluctuation suppressing in current study. At the same time, assume the maximum peak-to-peak amplitude of power fluctuation less than 500 [W].

2.3 Definition of Power Smoothing Precision In this paper, we define a smoothing precision to evaluate the smoothing level of the power fluctuation suppressing, as expressed in (1),

$$
\begin{aligned}
& \text { Smoothing Precision } \\
& =\frac{\text { Power Fluctuation of Lead }- \text { acid Battery }}{\text { Power Fluctuation of Natural Energy Power Supply }}
\end{aligned}
$$

Equation (1) indicates that there is the higher smoothing precision when the ratio is smaller. The ideal the power fluctuation suppressing is that the power fluctuation amplitude of lead-acid battery equals zero. That is the ratio of smoothing precision equals zero. Here, for the output voltage of battery is approximated constant, we consider the electric power smoothing by considering the output current smoothing. As a result, the smoothing precision also can be expressed by (2),

\section{Smoothing Precision \\ $=\frac{\text { Current Fluctuation of Lead }- \text { acid Battery }}{\text { Current Fluctuation of Natural Energy Power Supply }}$}

\section{Electric Power Smoothing Compensation Sys- tem}

\subsection{Conventional SNEPS with Electric Power Smooth-} ing Originally, EDLC is connected with battery in parallel directly as a hybrid energy-storage device of conventional SNEPS to compensate the power fluctuation by using the charge/discharge characteristics of EDLC, as shown in Fig. 3(a). Here, two of EDLCs are connected in parallel in experiment. The parameters of existing EDLC is, capacitance, $C_{E D}=7[\mathrm{~F}]$, the limited voltage, $V_{E D}=100[\mathrm{~V}]$. The inherent resistance of EDLC, $r_{E D}$ is considered in steady state and assumed as a constant. Here, the average value $r_{E D}=0.57[\Omega]$ in frequency characteristics test for $r_{E D}$ is adopted. In addition, assumes load as a constant impedance, $R=10[\Omega]$. The amplitude ratio of battery current and EDLC current is $r_{E D}: r_{B}$, that is about $2.85: 1$ when voltage of lead-acid battery, $V_{B}=24[\mathrm{~V}]$ and its inherent resistance, $r_{B}=0.2[\Omega]$. For example, when fluctuant current supply is, $i s=10 \sin (6 \pi t)+10[\mathrm{~A}]$, the operation result of electric power smoothing is shown in Fig. 3(b), the smoothing precision is $83.2 \%$. Fig. 3(b) shows, even though this circuit compensates a little part of power fluctuation, the effect of power fluctuation suppressing is not good. In this simple connection method of lead-acid battery and EDLC, power fluctuation is compensated only by the ratio of inherent resistance. Hence, a controllable electric power smoothing system is proposed by using a bi-directional Buck/Boost converter and EDLC to realize the desired compensation performance independent of the ratio of inherent resistance ${ }^{(7)}$. 


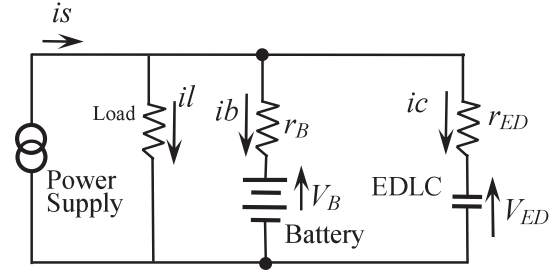

(a) System configuration

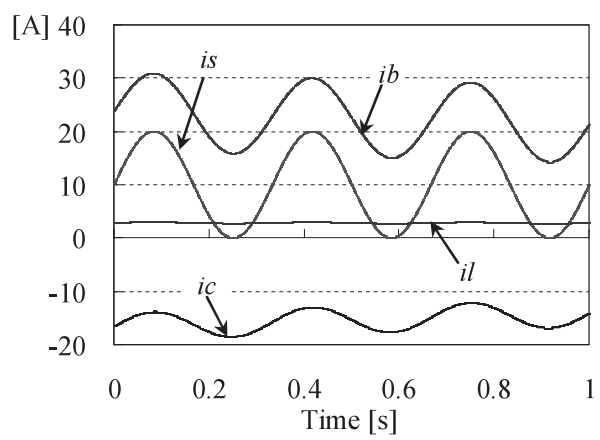

(b) Operation of electric power smoothing by EDLC $i s=10 \sin (6 \pi t)+10$ [A], Smoothing precision $=83.2 \%$

Fig. 3. Conventional SNEPS with electric power smoothing by EDLC

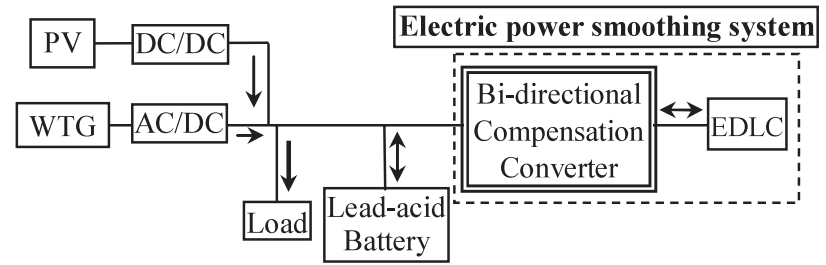

Fig. 4. SNEPS with proposed electric power smoothing system

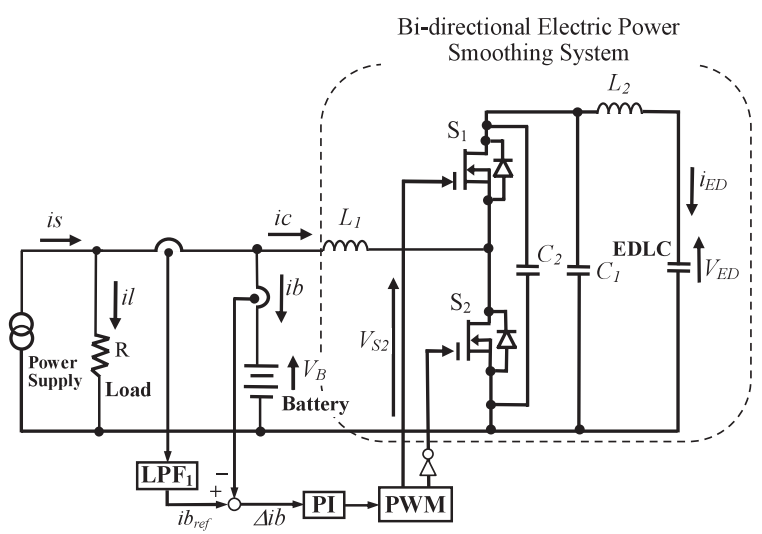

Fig. 5. Configuration of proposed electric power smoothing system

\subsection{Proposed Electric Power Smoothing System}

The proposed SNEPS with electric power smoothing system is shown in Fig. 4. Fig. 4 shows that EDLC connects with battery in parallel through a bi-directional compensation converter. We compensate the power fluctuation component of power supply by controlling the bi-directional compensation converter with EDLC and only supplies smoothed component to lead-acid battery.

Fig. 5 shows the configuration of the proposed electric power smoothing system. Table 1 shows the definition of the main variables of circuit. In this circuit, we regard the
Table 1. Definition of main variables of circuit

\begin{tabular}{|c|l|}
\hline Variables & \multicolumn{1}{c|}{ Meaning } \\
\hline$i s$ & Output current of power supply \\
\hline$i l$ & Load current \\
\hline$i b$ & Lead-acid battery current \\
\hline$V_{B}$ & Lead-acid battery voltage \\
\hline$i c$ & Compensation current of EDLC \\
\hline$V_{S 2}$ & Voltage of switch S2 \\
\hline$V_{C 1}$ & Voltage of capacitor, $C 1$ \\
\hline$i_{E D}$ & EDLC current \\
\hline$V_{E D}$ & EDLC voltage \\
\hline
\end{tabular}

power supply as a current source, and it represents the fluctuant power output of natural energy power system. Thus, the study purpose of this paper is to gain the smoothed current output for lead-acid battery. Load is assumed as constant impedance in current study. The bi-directional electric power smoothing system with EDLC is shown in the dashed line part of Fig. 5. Where, $C_{2}$ is a simple snubber of power switching device. In addition, a low-pass filter (LPF) consisting of $L_{2}$ and $C_{1}$ is used to protect EDLC from transient peak current. Because the service life of electro-chemical capacitor is short, here, a high frequency capacitor is adopted as $C_{1}$, $C_{1}$ can not be so large. Inductor $L_{2}$ is necessary evidently in order to protect the EDLC.

In control system, the DC and lower frequency components of current source is extracted through a low-pass filter $\left(\mathrm{LPF}_{1}\right)$ and selected as the reference, $i b_{r e f}$, of battery current, $i b$. Here, the cut-off frequency of $\mathrm{LPF}_{1}$ is selected as $0.1[\mathrm{~Hz}]$. We purpose to smooth the current fluctuation that its frequency is bigger than $0.1[\mathrm{~Hz}]$. In this paper, a proportional \& integral (PI) controller is adopted to control and adjust the steady state error $\Delta i b\left(\Delta i b=i b_{r e f}-i b\right)$ tend to zero. Switch frequency is $f_{s}=32.125[\mathrm{kHz}]^{(8)(9)}$. The voltage of switch S1 and switch S2 change by the duty factor. If the duty factor of switch $\mathrm{S} 2$ is assumed as $d$, ignoring the effect of inductor $L_{2}$, and regarding the voltage variety of $L_{2}$ as a part of modulate margin at the same time, then, $v_{C 1}=V_{E D}$. The voltage of switch S2 is expressed in (3),

$$
V_{S 2}=(1-d) V_{E D}
$$

Thus, the circuit operation is confirmed by comparing the voltage of $V_{S 2}$ and $V_{B}$. The fluctuant component is compensated through the charge/discharge of EDLC and battery current is smoothed.

3.3 Behavior of Proposed System In stable operation state, the proposed power smoothing system has the output as shown in Fig. 6(a). Here, a sine wave with a certain fluctuation frequency and DC component is assumed as the input of power supply and the fluctuation frequency of power supply is $f_{h}=3[\mathrm{~Hz}]$. Fig. 6(a) indicates the power fluctuation is greatly suppressed; the smoothing precision is only $2.2 \%$. Fig. 6(a) also indicates the AC component of power fluctuation is compensated and the constant component is supplied to lead-acid battery. Compares the simulation results of Fig. 6(a) with that of conventional SNEPS as shows in Fig. 3(b), the results indicate that the level of the power fluctuation suppressing can be regulated by parameters adjusting and control system in the proposed bi-directional electric power smoothing system.

However, LPF consisting of $L_{2}, C_{1}$ has resonance impact 
on system stability. When parameters change, vibration or saturation phenomenon often occurs on the part of current/voltage waveforms, as shown in Fig. 6(b). The vibration is caused by system instability which frequency is different from foundation fluctuation frequency of power supply. Such nonlinear phenomenon of partial output results in the analysis of the system characteristics which using conventional perturbation analysis theory becomes difficult. For the whole region of required power fluctuation, it becomes difficult to select a set of parameters to satisfy stability and smoothing at the same time.

Hence, the parameters that satisfy electric power smoothing output is selected in the stable region by using PSIM (Power Simulation) software and the effect trend-line. Where, the trend-line represents the effect of parameters on smoothing precision. In addition, because of the unstable phenomenon of output current, the peak-to-peak value of current, $i b_{p-p}$ is used to represent the current fluctuation of leadacid battery, and the peak-to-peak value of current source, $i s_{p-p}$ is used to represent the current fluctuation of natural energy power supply. In this case, smoothing precision is expressed in $\left(2^{\prime}\right)$.

$$
S=\frac{i b_{p-p}}{i s_{p-p}} .
$$

Considering the DC step variety of actual power supply that caused by the variety of natural source and the capacity of battery, we purpose to gain a smoothed electric power supply for lead-acid battery that smoothing precision $S \leq 10 \%$ in stable region. That is the current fluctuation of lead-acid

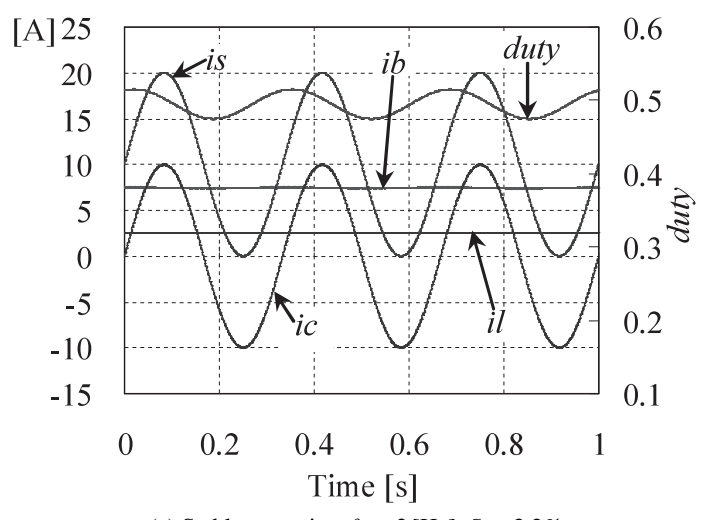

(a) Stable operation $f_{h}=3[\mathrm{~Hz}], S=2.2 \%$

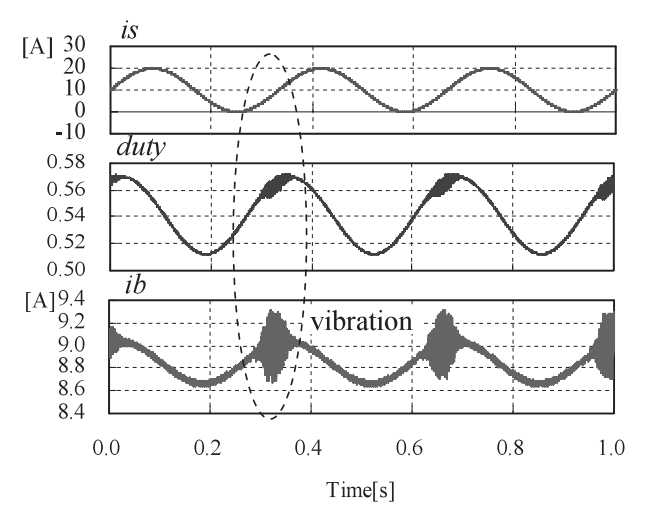

(b) Unstable phenomenon

Fig. 6. Behavior of the proposed electric power smoothing system battery is smaller than $10 \%$ of the current fluctuation of natural energy power supply.

\section{Parameter Selection of Proposed System}

To obtain the desired power smoothing performance, parameters determination is important during system design. The main parameters are initial voltage of EDLC, inductance of inductor $L_{1}$, inductance of inductor $L_{2}$, capacitance of condenser $C_{1}$, and proportional gain $K p$, integral gain $K i$ of PI controller. Here $C_{2}$ is determined by considering switching operation of circuit but isn't the issue, so, snubber $C_{2}$ with $C_{1}$ is considered together. In addition, the inherent resistance of $L_{1}, L_{2}$ and the wiring resistance, the ON resistance of FET are tested in experiment. They are expressed in following respectively, the inherent resistance of $L_{1}, r_{L 1}=0.04$ [ $\Omega$ ], the inherent resistance of $L_{2}, r_{L 2}=9.9[\mathrm{~m} \Omega]$, the $\mathrm{ON}$ resistance of FET containing the wiring resistance is $0.096[\Omega]$. These inherent resistances also are introduced into simulation.

4.1 Voltage Illustration of Steady State and $V_{E D}$ Selection The smoothing capability of steady state of proposed system is mainly considered in current study, but the system efficiency will be considered in future study. The average voltage waveform of steady state is shown in Fig. 7. Where, the switching harmonics are ignored. In proposed system, the voltage of inductor, $v_{L 1}$ and duty factor, $d$ varies along with the input fluctuant power variety. The Buck/Boost chopper of bi-directional converter operates alternately. The maximum variation in $v_{L 1}$ should be, $\Delta v_{L_{1}, \max }=V_{B}$ in order to prevent $d$ from minus. Thus, considering the voltage variation in $L_{2}, \Delta v_{L 2}, V_{E D}$ is selected according to (4).

The average duty factor is 0.5 in steady state.

$$
V_{E D} \geq 2 V_{B}
$$

Simultaneously, for 100 [V] of the limited voltage of existing EDLC, although the level of power fluctuation suppressing is higher when bigger $V_{E D}, V_{E D}=50[\mathrm{~V}]$ is selected within the controllable region in actual experiment when $V_{B}$ is 24 [V].

4.2 Parameters Selection using Trend-line Analysis in Simulation $\quad 0.5[\mathrm{~Hz}]$ and $10[\mathrm{~Hz}]$ are selected as two frequency example to make trend-line using PSIM simulation during parameters selection. Where, the trend-line indicates the effect of parameters on smoothing precision. Assuming $\Delta I_{S}=20$ [A], where $\Delta I_{S}$ is the amplitude of $\Delta i_{S}$. Parameters value usually is selected in the stable region where electric power smoothing can be obtained.

4.2.1 Inductor $L_{1}$ Selection In this paper, the inductor $L_{1}$ is chosen based on the assumption that the inductor

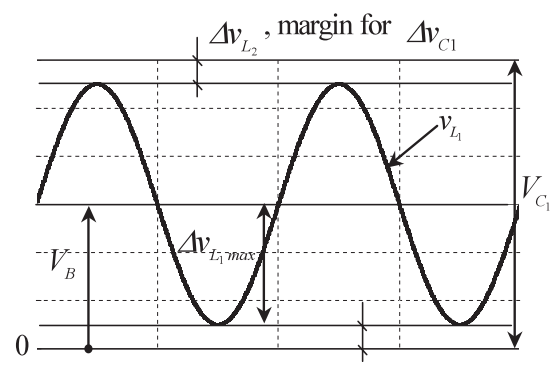

Fig. 7. Illustration of voltage variety 


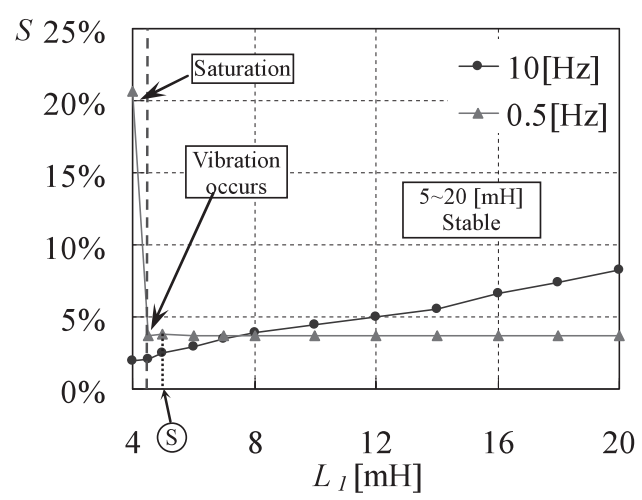

Fig. 8. Effect of inductor $L_{1}$ on smooth precision

ripple current is $\Delta I \leq 0.1$ [A], the required inductance $L_{1, \min }$ of inductor $L_{1}$ can be calculate from (5),

$$
L_{1} \geq L_{1, \min }=\frac{V_{E D}-V_{B}}{\Delta I} \cdot D \cdot T_{s}
$$

Where, duty factor is $D=0.5$, and $T_{s}$ is switch cycle, $T_{s}=31[\mu \mathrm{s}]$.

The $L_{1, \min }$ is calculated as $4.0[\mathrm{mH}]$. If assume the smaller inductor ripple current is assumed, the bigger $L_{1}$ is required.

In addition, the maximum of inductor $L_{1}$ can be calculated according to Fig. 7, as expressed as (6), (7).

$$
\begin{aligned}
& \Delta v_{L_{1}, \max }=\omega L_{1, \max } \cdot \Delta I_{S, \max } \\
& \Delta v_{L_{1}, \max } \leq V_{B} \ldots \ldots \ldots \ldots \ldots \ldots \ldots \ldots \ldots
\end{aligned}
$$

Where, $\omega=2 \pi f_{h}, f_{h}$ is the harmonic frequency of power fluctuation in the short period.

The value of $L_{1, \text { max }}$ changes along with frequency and amplitude of the fluctuant power. $L_{1, \text { max }}$ has a downward trend with growth in fluctuation frequency. Generally, the fluctuation frequency of short time focuses on the region of smaller than $10[\mathrm{~Hz}]$, when assume $f_{h}=10[\mathrm{~Hz}], \Delta I_{S, \text { max }}=20[\mathrm{~A}]$, the value of $L_{1, \max }$ is calculated as 19.1 [mH], thus, we select $L_{1}$ in region $4.0[\mathrm{mH}] \leq L_{1} \leq 19.1[\mathrm{mH}]$.

Fig. 8 shows the effect trend-line of $L_{1}$ on smoothing precision, $S$. Fig. 8 indicates that the value of $S$ has an upward trend with a growth in $L_{1}$. Though the range of stability extends when $L_{1}$ enlarge, but the charge/discharge response speed of EDLC becomes slow. Hence, we choose the smallest $L_{1}$ in the stable range, here, $L_{1}=5[\mathrm{mH}]$ is selected. The label (S) denotes the selected parameter. Similarly, the label, (S) is also shown in the following trend-line figures of parameters selection.

4.2.2 $L_{2}$ and $C_{1}$ Selection As mentioned above, $L_{2}$ and $C_{1}$ compose a LPF to protect EDLC from transient peak current. However, $L_{2}$ has great impact on system stability, the smaller $L_{2}$ the better system stability can be gained. Contrarily, the too smaller $L_{2}$ has more danger of transient peak current to EDLC. Hence, similar with $L_{1}$ selection, we select $L_{2}, C_{1}$ according to effect trend-line of $L_{2}, C_{1}$. Fig. 9 and Fig. 10 shows the effect trend-line of $L_{2}$ and $C_{1}$ respectively. Fig. 9 indicates that smoothing precision has an upward trend with a growth in $L_{2}$, especially when the frequency of power fluctuation is smaller. When the frequency of power fluctuation is $0.5[\mathrm{~Hz}]$, the vibration occurs at $L_{2}=0.6[\mathrm{mH}]$, and the vibration becomes bigger along with $L_{2}$ increases.

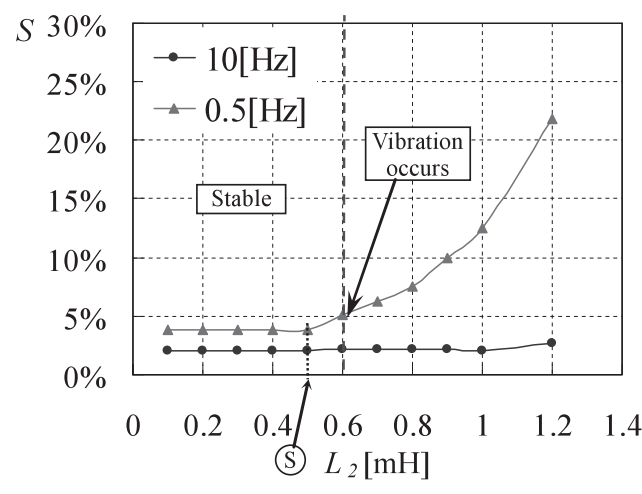

Fig. 9. Effect of $L_{2}$ on smooth precision $S$

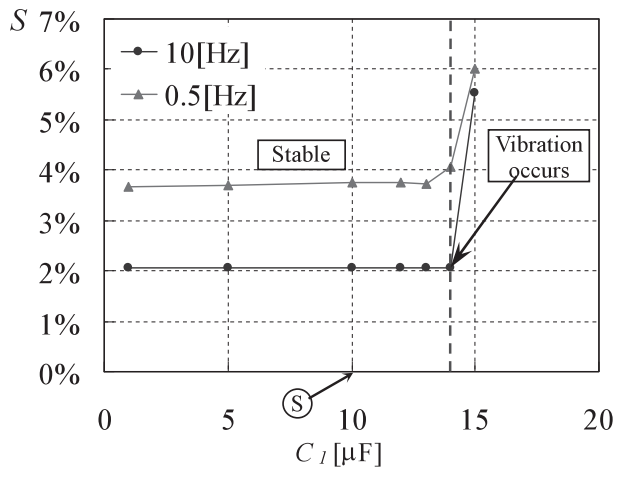

Fig. 10. Effect of $C_{1}$ on smooth precision $S$

When frequency of power fluctuation is $10[\mathrm{~Hz}]$, system is stable within the region of $L_{2}=0.1 \sim 1.2[\mathrm{mH}]$. Although smaller value of $L_{2}$ has the wider range of stability, transient peak current caused by switching flows into EDLC when $L_{2}$ is small too much and this is dangerous to EDLC. Hence, we select the largest $L_{2}=0.5[\mathrm{mH}]$ in stable range. The selection of $C_{1}$ is depended on the selected $L_{1}$ and the cutoff frequency, $f_{c}$ of LPF that consists of $L_{2}$ and $C_{1}$. Fig. 10 shows the trend-line of $C_{1}$. The vibration begins to occur at $C_{1}=14[\mu \mathrm{F}]$ when $C_{1}$ increases and vibration becomes bigger along with $C_{1}$ increases. Generally, cut-off frequency of LPF $\left(L_{2}, C_{1}\right)$ should be at least smaller than $10 \%$ of switching frequency in system design, that is $f_{c} \leq 3.2125[\mathrm{kHz}]$. Then, we can calculate the minimum of $L_{2} C_{1}$ from (8),

$$
f_{c}=\frac{1}{2 \pi \sqrt{L_{2} C_{1}}} \leq \frac{1}{10} f_{s} \ldots \ldots \ldots \ldots \ldots \ldots \ldots \cdots \cdots \cdots
$$

Here, $C_{1}=10[\mu \mathrm{F}]$ is selected according to actual electronic element. Then, the cut-off frequency, $f_{c}$ of LPF $\left(L_{2}\right.$, $C_{1}$ ) is $f_{c}=2.25[\mathrm{kHz}]$, it is smaller than $10 \%$ of switching frequency, $f_{s}=32.125[\mathrm{kHz}]$, and it is far higher than harmonic frequency of power fluctuation.

4.2.3 Selection of $\boldsymbol{K} \boldsymbol{p} \& \boldsymbol{K} \boldsymbol{i}$ of PI Controller The PI controller has following transfer function in (9),

$$
G_{(s)}=K p+K i / s
$$

Where, $K p$ is the proportional gain and $K i$ is the integral gain. Smoothing precision is controlled by tuning the $K p$ and $K i$ in stable region of system after selected $L_{1}, L_{2}$ and $C_{1}$. Proportional gain, $K p$ has a deep effect to the stability of system and the smoothing precision. The effect trend-line $K p$ on $S$ is shown in Fig. 11(a). Fig. 11(a) indicates that $S$ 
has a downward trend with growth in $K p$, especially when power fluctuation frequency is higher. The smoothing precision of $10[\mathrm{~Hz}]$ changes bigger than that of $0.5[\mathrm{~Hz}]$ along with the growth of $K p$ under the same condition. The smaller smoothing precision value can be gained when $K p$ is larger. However, the vibration occurs when $K p$ is too large. Contrarily, the stable range is wider if $K p$ is smaller. However, the following speed to reference becomes slow if the $K p$ is small too much. In addition, the effect of the power fluctuation suppressing is worse even though there has not vibration phenomena in lower $K p$. Hence, we select the largest $K p$ in the stable range and to ensure a smaller smoothing precision $S$. Here, we select $K p=0.3$ [1/A].

Fig. 11(b) shows the effect trend-line of integral gain, $K i$ on $S$. Even though Fig. 11(b) shows that $K i$ has little influence on the system stability and the smoothing precision during $K i=0.002 \sim 200$ [1/As], integral control is still necessary. Integral control is adopted mainly to adjust the steady state error of output current, $i b$ to zero. Additional, the response speed of PI should be faster than that of $\mathrm{LPF}_{1}$ according to the response characteristics of system. That is the damping time constant of PI controller, $\tau_{P I}$ should be smaller than that of $L_{P F}, \tau_{L P F_{1}} . \tau_{P I}$ and $\tau_{L P F_{1}}$ are expressed in (10) and (11) respectively. $\tau_{L P F_{1}}=1.59[\mathrm{~s}]$ when the cut-off frequency of $\mathrm{LPF}_{1}$ is $0.1[\mathrm{~Hz}]$. Thus, $K i$ should be:

$$
\frac{K p}{K i} \leq 1.59[s], \text { that is } K i \geq 0.19 \text { when } K p=0.3[1 / \mathrm{A}] .
$$

We select the smaller $K i, K i=0.2[1 / \mathrm{A} \cdot \mathrm{s}]$ that satisfy above requirement in the stability region, here, $\tau_{P I}=1.5[\mathrm{~s}]$. Table 2 shows the selected parameters.

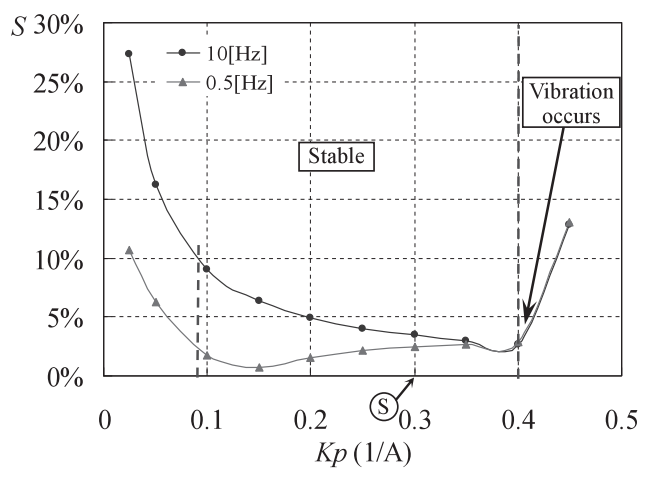

(a) Effect of parameters $K p$ on $S$

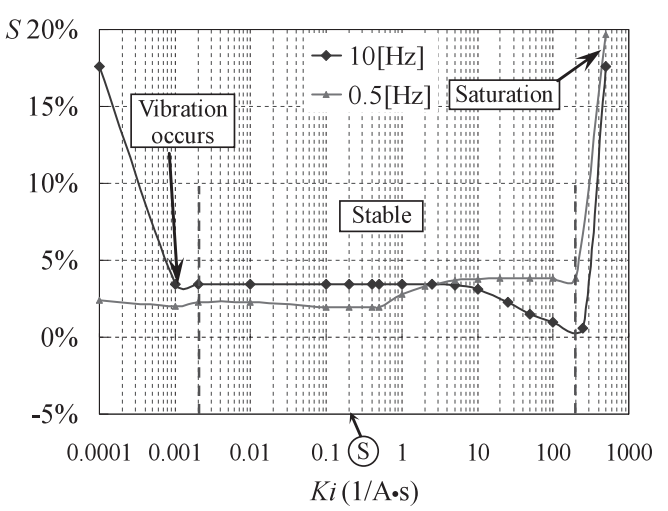

(b) Effect of parameters $K i$ on $S$

Fig. 11. Effect of parameters $K p$, and $K i$ on $S$

$$
\begin{gathered}
\tau_{L P F_{1}}=\frac{1}{\omega}=\frac{1}{2 \pi f_{1}} \\
\tau_{P I}=\frac{K p}{K i} \cdots \ldots \ldots
\end{gathered}
$$

For general condition, the new system also can be designed to maintain the power smoothing performance based on the known system parameters if natural condition or energy storage requirement changes. Parameters can be modulated by considering the unitary variety of voltage, current and impedance. Because of limited pages, the detail of procedure do not present in this paper.

4.3 Characteristic Analysis in Low Frequency Region

4.3.1 State-space Average Model There are two operation modes in the proposed bi-directional Buck/Boost compensation converter. In mode $1, \mathrm{~S} 1$ is open and $\mathrm{S} 2$ is closed. In mode $2, \mathrm{~S} 1$ is closed and S2 is open ${ }^{(10)}$. The two operation "modes' are shown as state transition diagram in Fig. 12. The capacitance of EDLC is so big that EDLC is assumed as a voltage source in following state analysis, except for extremely lower frequency region.

The simple application of nodal and mesh equations to the topology, the state-space model equation of mode 1 and mode 2 are gained, as shown in (12) and (13) respectively. Here, $\dot{x}(t)$ and $x(t)$ are the state vector and the state variable as shown in (14) and (15) respectively, where, the state variables $i c(t), i_{E D}(t)$ and $v_{C 1}(t)$ denote the variation of AC current that flows into $L_{1}$, the current of EDLC and the voltage of $C_{1}$, respectively. $u(t)$ in (16) represents the input variables, including power source current $i s(t)$, the battery voltage $V_{B}$ and the EDLC voltage $V_{E D}$. In addition, the output, $y(t)$ in

Table 2. Selected Parameters and value

\begin{tabular}{|c|c|}
\hline Parameters & Value \\
\hline$V_{E D}$ & $50[\mathrm{~V}]$ \\
\hline$L_{1}$ & $5[\mathrm{mH}]$ \\
\hline$L_{2}$ & $0.5[\mathrm{mH}]$ \\
\hline$C_{1}$ & $10[\mu \mathrm{F}]$ \\
\hline$K p$ & $0.3[1 / \mathrm{A}]$ \\
\hline$K i$ & $0.2[1 / \mathrm{A} \cdot \mathrm{s}]$ \\
\hline
\end{tabular}

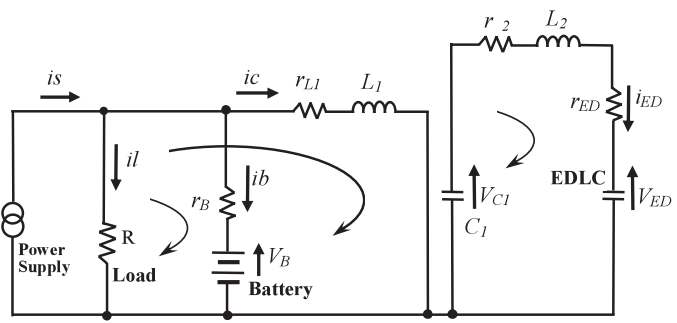

(1) Mode 1, S1: OFF, S2: ON

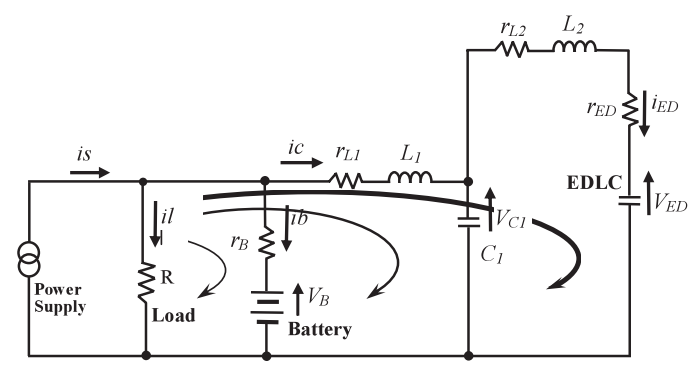

(2) Mode 2, S1: ON, S2: OFF

Fig. 12. State-space model 
(17) is the battery current $i b(t)$.

(1) Mode 1

$$
\left\{\begin{array}{l}
\dot{x}(t)=A_{1} x(t)+B_{1} u(t) \\
y(t)=C_{1} x(t)+D_{1} u(t)
\end{array}\right.
$$

(2) Mode 2

$$
\begin{aligned}
& \left\{\dot{x}(t)=A_{2} x(t)+B_{2} u(t)\right. \\
& y(t)=C_{2} x(t)+D_{2} u(t) \\
& \dot{x}(t)=\left[\begin{array}{lll}
\frac{d i c}{d t} & \frac{d i_{E D}}{d t} & \frac{d v_{c 1}}{d t}
\end{array}\right]^{T} \\
& x(t)=\left[\begin{array}{lll}
i c(t) & i_{E D}(t) & v_{C 1}(t)
\end{array}\right]^{T} \\
& u(t)=\left[\begin{array}{lll}
i s(t) & V_{B} & V_{E D}
\end{array}\right]^{T} \\
& y(t)=i b(t)
\end{aligned}
$$

By averaging the state equations over one period, assuming duty factor of switch $\mathrm{S} 2$ is $d$, then, the state-space average model is gained as shown in (18).

$$
\left\{\begin{array}{l}
\dot{x}(t)=\left[A_{1} d+A_{2}(1-d)\right] x(t)+\left[B_{1} d+B_{2}(1-d)\right] u(t) \\
y(t)=\left[C_{1} d+C_{2}(1-d)\right] x(t)+\left[D_{1} d+D_{2}(1-d)\right] u(t)
\end{array}\right.
$$

Or:

$$
\left\{\begin{array}{l}
\dot{x}(t)=A x(t)+B u(t) \\
y(t)=C x(t)+D u(t)
\end{array}\right.
$$

Where coefficient matrices of A, B, C and D are expressed in (19), (20), (21) and (22) respectively.

$$
\begin{aligned}
& A=\left[\begin{array}{ccc}
-\frac{r_{L_{1}}\left(R+r_{B}\right)+R \cdot r_{B}}{L_{1}\left(R+r_{B}\right)} & 0 & -\frac{1-d}{L_{1}} \\
0 & -\frac{r_{L_{2}}+r_{E D}}{L_{2}} & \frac{1}{L_{2}} \\
\frac{1-d}{C_{1}} & -\frac{1}{C_{1}} & 0
\end{array}\right] \\
& \text {............... (19) } \\
& B=\left[\begin{array}{ccc}
\frac{R \cdot r_{B}}{L_{1}\left(R+r_{B}\right)} & \frac{R}{L_{1}\left(R+r_{B}\right)} & 0 \\
0 & 0 & -\frac{1}{L_{2}} \\
0 & 0 & 0
\end{array}\right] \\
& C=\left[\begin{array}{lll}
-\frac{R}{R+r_{B}} & 0 & 0
\end{array}\right] \\
& D=\left[\begin{array}{lll}
\frac{R}{R+r_{B}} & -\frac{1}{R+r_{B}} & 0
\end{array}\right]
\end{aligned}
$$

The infinitesimal differentials rather than finite differences is applied to analyze the proposed converter. To assume nonlinear effect is small, and it would not affect the overall accuracy of analysis significantly, but would effectively linearize the system. Transfer functions are gained according above analysis.

4.3.2 Frequency Characteristics The block diagram of the proposed system is derived as shown in Fig. 13, where, $G_{1}(\mathrm{~s}), G_{22}(\mathrm{~s})$ and $G_{3}(\mathrm{~s})$ are 2-nd order low-pass filters (LPFs). $G_{1}(\mathrm{~s})$ is the transfer function of $\mathrm{LPF}_{1}$ that extracts the DC and lower frequency components of fluctuant power as the reference of battery current. The battery current, $i \hat{b}$ consists of two parts. One part comes from the power fluctuation directly, as shown in Fig. 13 . Here, $G_{5}(\mathrm{~s})$ indicates the transfer function of from the output current (input) $\hat{i s}$ of power fluctuation supply that supplies to the electric power smoothing system to the battery current (output) $\hat{i b}$ for small signals, which is compensated by another part of $\hat{i b}$ from the duty factor $\hat{d}$, where $G_{0}(\mathrm{~s})$ is the transfer function of from duty cycle $\hat{d}$ to battery current (output) $\hat{i b}$. In Fig. 13, the feedback gain, $\mathrm{H}$ is 1 . Then, close-loop transfer function $H_{c}(\mathrm{~s})$ from the input $\hat{i s}$ to the output $\hat{i b}$ is expressed in (23),

$$
H_{c}(s)=\frac{\hat{i}(s)}{\hat{i}(s)}=-\frac{G_{5}(s)}{G_{a 2}(s)} \cdot G_{1}(s)+\frac{G_{a 2}(s)}{1+G_{a 2}(s) \cdot H(s)}
$$

Where,

$$
G_{a 2}(s)=\left(G_{22}(s) \cdot K p+\frac{K i}{s}\right) \cdot G_{3}(s) \cdot K_{c} \cdot G_{0}(s)
$$

$K_{c}$ is the proportional coefficient of the comparator. Here, $K_{c}$ is set at $1 / 20$.

The calculation results of frequency characteristics of system are shown in Fig. 14, where duty factor is $d=0.5$. Duty factor, $d$ changes larger in the case of larger power fluctuation, the damping factor from the output current of power supply (input) $\hat{i s}$ to the battery current (output) $\hat{i b}$ varies with the frequency of power fluctuation, and arrives the lowest around $0.7[\mathrm{~Hz}]$. Here, the damping factor is adopted to express the smoothing precision, $S$ and evaluate the level of power fluctuation suppressing when the instability phenomenon does not occur, as shown in (25). When the damping factor is smaller than $-20[\mathrm{~dB}]$, the smoothing precision is smaller than $10 \%$.

$$
\text { damping factor }=20 \log _{10}\left|H_{c}(j \omega)\right|
$$

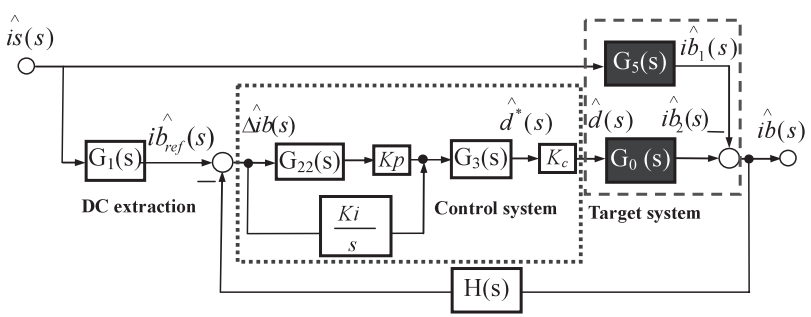

Fig. 13. Block diagram of proposed electric power smoothing system

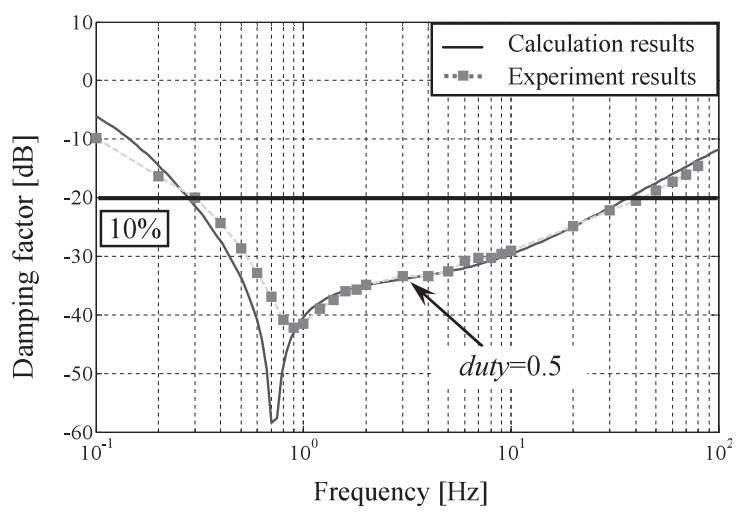

Fig. 14. Frequency characteristics 
The frequency characteristics of Fig. 14 indicates that power fluctuation is damped smaller than $-20[\mathrm{~dB}]$ in the target region of $0.5[\mathrm{~Hz}] \leq f_{h} \leq 3[\mathrm{~Hz}]$. This result satisfies the requirement of smoothing precision, $S<10 \%$. Experimental results of the frequency characteristics is shown in Fig. 14 too. Fig. 14 indicates the frequency characteristics results of experiment and calculation correspond comparatively well. Power fluctuation suppression and smooth electric power supply can be realized in actual system.

\section{Experimental Results}

In order to further validate the feasibility of proposed power smoothing system in actual natural power system, the proposed system is introduced into actual wind power system. The rated power of existing wind turbine generator (WTG) is 400 [W], lead-acid battery voltage, $V_{B}$ is $24[\mathrm{~V}]$, Load is constant, $\mathrm{R}=10[\Omega]$. Other parameters are shown in Table 2.

Fig. 15(a) shows an example of experimental results. Fig. 15 (a) shows the proposed system avoid the instantaneous peak current and over charge of lead-acid battery when there has transient step output of WTG power system. EDLC compensates alternative component of power fluctuation by charge/discharge. At the same time, Fig. 15(a) indicates that the smoothed component is supplied to lead-acid battery by introducing the proposed system. The power fluctuation of WTG output is suppressed greatly. The electric power smoothing output is benefit to decreasing the recharge cycles times of lead-acid battery. The recharge cycle times can

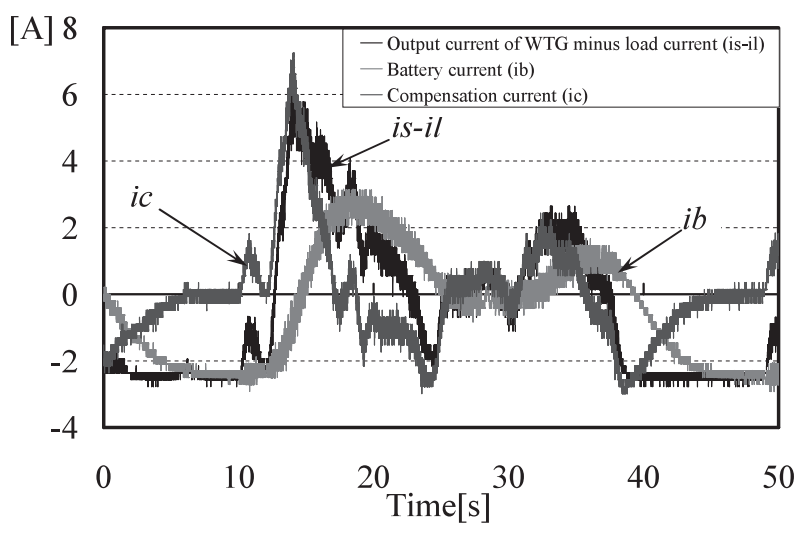

(a) Experiment results in actual wind power system

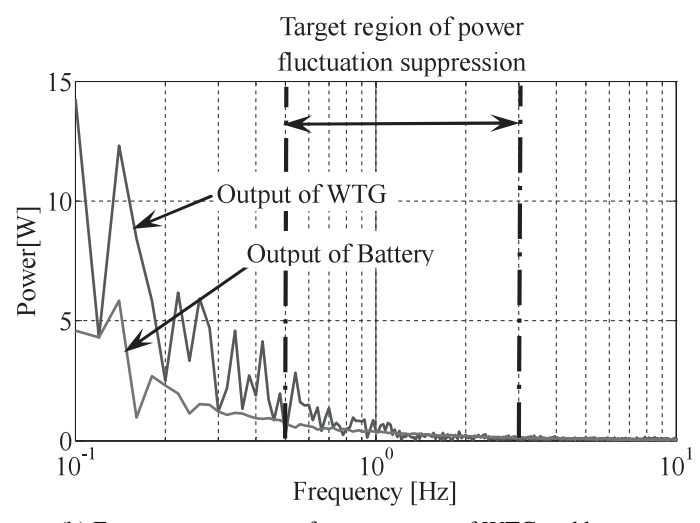

(b) Frequency spectrum of output power of WTG and battery

Fig. 15. An example of stand-alone WTG output with proposed system be further decreased if the battery current is controlled not to reach zero. For example, if a control is added to control the battery current not reach zero in the period 25 [s] $<$ Time $<$ 32 [s] of Fig. 15(a), the charge/discharge times will be decreased near $i b=0$ and the service life also be prolonged greatly. These will be the further task of our study in the future. Fig. 15(b) shows the frequency spectrum of power output of WTG and battery. Fig. 15(b) also indicates that fluctuant power of WTG is suppressed effectively by using the proposed system. The experimental results verified the selected parameters are useful for smoothing the power fluctuation in the frequency target region. The usefulness of the short period power fluctuation suppressing of the proposed system is confirmed by the experimental results.

\section{Conclusion}

An electric power smoothing system for lead-acid battery of SNEPS using bi-directional compensation converter and EDLC is proposed in this paper. The validity of the proposed system is confirmed by the frequency characteristics analysis of calculation and experimental results. And the feasibility of proposed system also be verified in actual WTG power system. The fluctuant power is suppressed effectively and smoothed power is supplied to lead-acid battery successfully by introducing the proposed electric power smoothing system. The smoothing power supply is benefit for prolonging the service life of lead-acid battery. Furthermore, the proposed electric power smoothing system is applicable for different SNEPSs under general natural condition ${ }^{(11)}$.

(Manuscript received Nov. 10, 2006, revised July 19, 2007)

\section{References}

( 1 ) Y.-S. Jung, J.-Y. Lee, and M.-J. Youn: “A New Small Signal Modeling of Average Current Mode Control", Power Electronic Specialists Conference, PESE 98 Record 29th Annual IEEE, Vol.2, pp.1118-1124 (1998)

( 2 ) The National Renewable Energy Laboratory: "Small Wind Energy Systems for the Homeowner", Department of Energy, pp.1-6, U.S (1997-1)

( 3 ) E. Tamura, and Y. Matsuda etc.: "The advanced of supercapacitor-Electric Double Lay Capacitor", Function chemical series of electron and ion, Vol.2, ENE.DHI. ESU., pp.305-327 (2002-1)

(4) H. Kaneuchi, T. Yachi, M. Nitta, and T. Tani: "The Construction of a Wind Turbine System with an EDLC for Hydrogen Production", Wind power generation, the 2005 International Power Electronics conference, pp.1085-1092 (2005)

( 5 ) G.E. Mayer: "Overview on batteries and charging methods", in Proceeding High Frequency Power Converter Conference, pp.205-214 (1994)

( 6 ) N. Ushiki, K. Fujita, and T. Nakano: "A Suppression of Power Fluctuations by using EDLCS in Variable Speed Wind Power Generation System", Power and Energy Systems, EuroPES (2003)

( 7 ) T. Takano: "Natural Energy Power and Energy Storing Technology", IEEJ Transactions on Power and Energy, Vol.126, No.9, pp.857-860 (2006)

( 8 ) G.A. Papafotiou and N.I. Margaris: "calculation and stability Investigation of Periodic Steady State of the Voltage Controlled Buck DC-DC Converter", IEEE Trrans. Power Electron., Vol.19, No.4, pp.959-970 (2004-7)

( 9 ) M.D. Bernardo, F. Garofalo, L. Glielmo, and F. Vasca: "Switchings bifurcations ans chaos in dc/dc converters", IEEE Trans. Circuits Syst. I, Vol.45, pp.133-141 (1998-2)

(10) A. Allison and D. Abbott: "Some Benefits of Random Varaibles in Switched Control System", Microelectro. Journal 31, pp.515-522 (2000)

(11) Q.-G. Wang, T.-H. Lee, H.-W. Fung, Q. Bi, and Y. Zhang: IEEE Trrans. Contr. Syst. Technol., Vol.7, No.4, pp.457-465 (1999-7) 
Yan Jia (Student Member) was born in Inner Mongolia, China. She

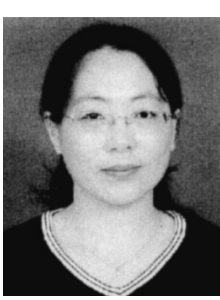
received the B.Sc. and M. Sc. degree in energy and power engineering from the Inner Mongolia University of Technology, China, in 1996 and 2001 respectively. She is currently pursuing the Ph.D. degree at Mie University, Japan. Her main research in past includes heat energy conversion and exhaust gas recycle, hybrid renewable power system configuration. Currently, her main research includes electric energy storage and electric power smoothing control of Stand-alone Natural Energy Power System.

Ryosuke Shibata (Student Member) He was born in Hyogo, Japan

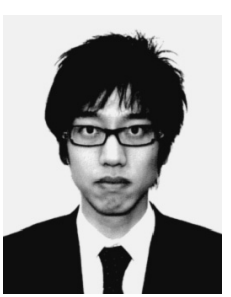
on Jan. 11, 1982. He received the B.Sc. degree from Energy System Lab, Dept. of E.E., Mie University in 2006. He is currently in Master Course at Inf. Sys. Lab, Dept. of E.E., Nagoya Univ. In Mie Univ., His main research includes the study of power smoothing system by using EDLC for small stand-alone power system.
Naoki Yamamura (Member) was born in Mie, Japan on October 20,

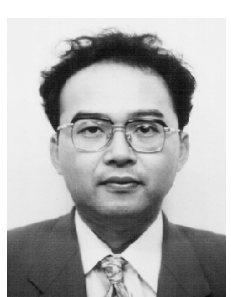
1964. He received the B.Sc., degree M.Sc. degree and Dr. Eng. degree from Nagoya Institute of Technology in 1989, 1991 and 1993, respectively, all in Electrical Engineering. Since 1993, he has been associated with the Department of Electrical Intelligence Engineering, Nagoya Institute of Technology, where he was an Assistant Researcher from 1993. Since 1998, he has been associated with the Department of Electrical and Electronic Eng., Mie University, where he is an Assistant Professor from 1998. His present research is the development of power generation system using natural energy, active filter and Fuel-cell generation system. Dr. Yamamura is a member of the IEEE, JIPE and IEE of Japan.

Muneaki Ishida (Member) was born in Aichi, Japan on April 12,

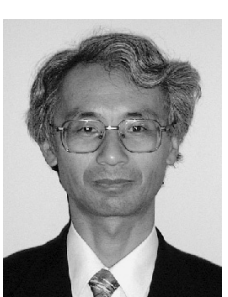
1952. He received the B.Sc. degree, M.Sc. degree and Dr. Eng. degree from Nagoya University in 1975 , 1977 and 1980, respectively, all in Electrical and Electronic Engineering. He was with Nagoya University as a Research Associate in the Department of Electrical Engineering from 1980 to 1987. Since 1987, he has been with Mie University, initially as an Associate professor and from Oct., 1996 as a Professor. He is engaged in research on static power converters, AC motor drive system, power generation system using natural energy 